\title{
ON AN EQUIVALENCE OF FUZZY SUBGROUPS III
}

\author{
V. MURALI and B. B. MAKAMBA
}

Received 29 May 2002

\begin{abstract}
This paper is the third in a series of papers studying equivalence classes of fuzzy subgroups of a given group under a suitable equivalence relation. We introduce the notion of a pinned flag in order to study the operations sum, intersection and union, and their behavior with respect to the equivalence. Further, we investigate the extent to which a homomorphism preserves the equivalence. Whenever the equivalences are not preserved, we have provided suitable counterexamples.
\end{abstract}

2000 Mathematics Subject Classification: 20N25, 20D30, 03E72, $20 \mathrm{~F} 22$.

1. Introduction. For the benefit of the reader and also to fix notations, we recall the following from [3].

We use $\mathbf{I}=[0,1]$, the real unit interval, as a chain with the usual ordering in which $\wedge$ stands for infimum (or intersection) and $\vee$ stands for supremum (or union).

A fuzzy subset of a set $X$ is a mapping $\mu: X \rightarrow \mathbf{I}$. The union, intersection of two fuzzy sets, and complementation of a fuzzy set are defined using sup pointwise, inf pointwise, and $1-\mu$ operator pointwise, respectively. We denote the set of all fuzzy subsets of $X$ by $\mathbf{I}^{X}$. Further, we denote fuzzy sets by the Greek letters $\mu, v, \eta$, and so forth. If $X$ is a finite group (which is what we will be interested in this paper), a fuzzy set $\mu$ is said to be a fuzzy subgroup if $\mu(x+y) \geq \mu(x) \wedge \mu(y)$ for all $x, y \in X$ and $\mu(x)=\mu(-x)$. Without loss of generality, we assume that $\mu(0)=1$. From this assumption, we notice that the only admissible fuzzy subgroup of the trivial group is $\mu(0)=1$.

We define an equivalence relation $\sim$ on $\mathbf{I}^{X}$ as follows: $\mu \sim \nu$ if and only if

(i) for all $x, y \in X, \mu(x)>\mu(y)$ if and only if $v(x)>v(y)$,

(ii) $\mu(x)=0$ if and only if $\nu(x)=0$.

It is easily checked that this relation is indeed an equivalence relation on $\mathbf{I}^{X}$ and when restricted to $2^{X}$, this relation coincides with an equality of sets. We denote this equivalence relation by $\mu \sim v$. Originally, this idea of equivalence was prompted by $[3,4,5]$.

NoTE 1.1. The condition, $\mu(x)=0$ if and only if $\nu(x)=0$, simply says that the supports of $\mu$ and $\nu$ are equal, where by support we mean $\operatorname{supp} \mu=\{x \in$ $X: \mu(x)>0\}$. The above condition cannot be made redundant since it is an essential part of the equivalence relation. 
Throughout this paper, by a group $G$ we mean a finite group. By a flag we mean a chain of subgroups of the form $0 \subset G_{1} \subset G_{2} \subset \cdots \subset G_{n}=G$. For some examples of flags used in this paper, we refer to [1]. In [1], flags were referred to as series.

We recall from [4] that a keychain $l$ means a set of real numbers in $\mathbf{I}$ of the form $1 \geq \lambda_{1} \geq \lambda_{2} \geq \cdots \geq \lambda_{n} \geq 0$, where the $\lambda_{i}$ 's are not all necessarily distinct. The $\lambda_{i}$ 's are called pins. By a pinned flag, we mean a pair $(\mathscr{C}, l)$, of a flag $\mathscr{C}$ and a keychain $l$, written as follows:

$$
0^{1} \subset G_{1}^{\lambda_{1}} \subset G_{2}^{\lambda_{2}} \subset \cdots \subset G_{n}^{\lambda_{n}}
$$

We associate the following fuzzy subgroup with such a pinned flag $(\mathscr{C}, l)$ :

$$
\mu(x)= \begin{cases}1, & x=0, \\ \lambda_{1}, & x \in G_{1} \backslash\{0\}, \\ \lambda_{2}, & x \in G_{2} \backslash G_{1}, \\ \vdots & \\ \lambda_{n}, & x \in G_{n} \backslash G_{n-1},\end{cases}
$$

where the component $G_{n}$ is the whole group $G$. We denote this simply by $G_{n}{ }^{\lambda_{n}}=G^{\lambda_{n}}$. That $\mu$ is indeed a fuzzy subgroup on $G$ may be quickly verified using the definition. In this case we say that $\mu$ is represented by the pinned flag

$$
0^{1} \subset G_{1}^{\lambda_{1}} \subset G_{2}^{\lambda_{2}} \subset \cdots \subset G_{n}^{\lambda_{n}}
$$

Conversely, every fuzzy subgroup $\mu$ may be decomposed into a pinned flag as above by considering suitable $\alpha$-cuts. For further details, see [3, 4]. Similar techniques have been used in [2].

2. Homomorphisms and equivalences. In this section, given a homomorphism between two groups, we look at the equivalence classes of homomorphic images and preimages of fuzzy subgroups. Firstly, we recall that if $f: G \rightarrow H$ is a homomorphism, by $f(\mu)$ we mean the image of a fuzzy subset $\mu$ of $G$ and it is a fuzzy subset of $H$ defined by, for $h \in H,(f(\mu))(h)=\sup \{\mu(g)$ : $g \in G, f(g)=h$ \} if $f^{-1}(h) \neq \varnothing$ and $f(\mu)(h)=0$ if $f^{-1}(h)=\varnothing$. Similarly, if $v$ is a fuzzy subset of $H$, the preimage of $f^{-1}(v)$ is a fuzzy subset of $G$ and is defined by $\left(f^{-1}(v)\right)(g)=v(f(g))$. The subgroup property is transferred to images and preimages by a homomorphism between groups. For further properties of images and preimages of fuzzy sets under a mapping, see [6]. Throughout this section, we suppose that $f: G \rightarrow H$ is a homomorphism from a group $G$ to $H$.

Proposition 2.1. If $\mu \sim \nu$, then $f(\mu) \sim f(\nu)$. 
Proof. Clearly, supp $\mu=\operatorname{supp} v$ implies $f(\operatorname{supp} \mu)=f(\operatorname{supp} v)$, which in turn implies supp $f(\mu)=\operatorname{supp} f(\nu)$. For the other part, let $x, y \in H$ such that $f(\mu)(x)>f(\mu)(y)$. It is enough to show that $f(\nu)(x)>f(\nu)(y)$. Now, there is an $x^{\prime} \in G$ such that $f\left(x^{\prime}\right)=x$ and $\mu\left(x^{\prime}\right)>\mu\left(y^{\prime}\right)$ for all $y^{\prime} \in G$ such that $f\left(y^{\prime}\right)=y$. Since $\mu \sim v, v\left(x^{\prime}\right)>v\left(y^{\prime}\right)$. This in turn implies $f(v)(x)>f(v)(y)$. Thus, $f(\mu) \sim f(\nu)$.

It is straightforward to check that if $\mu \sim v$ in $H$, then $f^{-1}(\mu) \sim f^{-1}(v)$ in $G$. Also, one could consider the behavior of inequivalent fuzzy subgroups under a homomorphism. In general, one would expect inequivalent fuzzy subgroups to have inequivalent images, but the following example illustrates that two inequivalent fuzzy subgroups may have equivalent images under a homomorphism.

For the next two examples, consider $\mathbb{Z}_{6}=\{0,1,2,3,4,5\}$ as a given group and $f: \mathbb{Z}_{6} \rightarrow \mathbb{Z}_{6}$ defined by $f(1)=2$ as a given homomorphism.

EXAMPLE 2.2. Let

$$
\mu(x)=\left\{\begin{array}{ll}
1, & x=0, \\
\frac{1}{2}, & x \in \mathbb{Z}_{2} \backslash 0, \\
\frac{1}{4}, & \text { otherwise, }
\end{array} \quad v(x)= \begin{cases}1, & x=0, \\
\frac{1}{2}, & x \in \mathbb{Z}_{3} \backslash 0, \\
\frac{1}{4}, & \text { otherwise. }\end{cases}\right.
$$

Clearly, $\mu$ is inequivalent to $v$. But

$$
f(\mu)(x)=\left\{\begin{array}{ll}
1, & x=0, \\
\frac{1}{4}, & \text { otherwise, }
\end{array} \quad f(v)(x)= \begin{cases}1, & x=0, \\
\frac{1}{2}, & \text { otherwise }\end{cases}\right.
$$

which are clearly equivalent.

Similarly, we may have inequivalent fuzzy subgroups giving rise to equivalent preimages under a homomorphism.

EXAMPLE 2.3. Let

$$
\mu(x)=\left\{\begin{array}{ll}
1, & x=0, \\
\frac{1}{2}, & x \in \mathbb{Z}_{2} \backslash 0, \\
\frac{1}{3}, & \text { otherwise, }
\end{array} \quad v(x)= \begin{cases}1, & x=0, \\
\frac{1}{2}, & x \in \mathbb{Z}_{3} \backslash 0, \\
\frac{1}{3}, & \text { otherwise. }\end{cases}\right.
$$

Clearly, $\mu$ is inequivalent to $v$. But

$$
f^{-1}(\mu)(x)=\left\{\begin{array}{ll}
1, & x=0, \\
\frac{1}{3}, & \text { otherwise, }
\end{array} \quad f^{-1}(v)(x)= \begin{cases}1, & x=0, \\
\frac{1}{2}, & \text { otherwise }\end{cases}\right.
$$

which are equivalent. 
It is clear that if $f: G \rightarrow H$ is an epimorphism, then $f\left(f^{-1}(\mu)\right)=\mu$; therefore, if $\mu$ and $\nu$ are inequivalent fuzzy subgroups of $H$, then $f^{-1}(\mu)$ and $f^{-1}(\nu)$ are inequivalent fuzzy subgroups of $G$. Similarly, it is clear that if $f: G \rightarrow H$ is a monomorphism, then $f^{-1}(f(\mu))=\mu$; therefore, if $\mu$ and $v$ are inequivalent fuzzy subgroups of $G$, then $f(\mu)$ and $f(\nu)$ are inequivalent fuzzy subgroups of $H$. Therefore, equivalences and unequivalences of fuzzy subgroups are preserved under an isomorphism, but the notion of a fuzzy isomorphism is very different (see [3]).

3. Equivalences under operation. In general, the operations of infimum (the intersection), supremum (the union), and sum of fuzzy subgroups need not preserve the equivalence classes of fuzzy subgroups. We have the following example.

EXAMPLE 3.1. Suppose that $\mu \sim v$ and $\mu^{\prime} \sim v^{\prime}$. Then it is not necessary that $\mu \wedge \mu^{\prime} \sim v \wedge v^{\prime}$.

Let $G$ be the group of integers $\mathbb{Z}$ under addition.

Let

$$
\begin{array}{ll}
\mu(x)= \begin{cases}1, & x \in 2 \mathbb{Z}, \\
\frac{1}{3}, & \text { otherwise, }\end{cases} & \mu^{\prime}(x)= \begin{cases}1, & x \in 2 \mathbb{Z}, \\
\frac{1}{10}, & \text { otherwise, }\end{cases} \\
\nu(x)= \begin{cases}1, & x \in 3 \mathbb{Z}, \\
\frac{1}{2}, & \text { otherwise, }\end{cases} & v^{\prime}(x)= \begin{cases}1, & x \in 3 \mathbb{Z}, \\
\frac{1}{20}, & \text { otherwise. }\end{cases}
\end{array}
$$

Then

$$
(\mu \wedge \nu)(x)= \begin{cases}1, & x \in 6 \mathbb{Z}, \\
\frac{1}{2}, & x \in 2 \mathbb{Z} \backslash 6 \mathbb{Z}, \quad\left(\mu^{\prime} \wedge v^{\prime}\right)(x)=\left\{\begin{array}{ll}
1, & x \in 6 \mathbb{Z}, \\
\frac{1}{10}, & \text { otherwise, }
\end{array} \quad x \in 3 \mathbb{Z} \backslash 6 \mathbb{Z},\right. \\
\frac{1}{20}, & \text { otherwise. }\end{cases}
$$

Firstly, we notice that

$$
\mu \sim \mu^{\prime}, \quad v \sim v^{\prime}
$$

Secondly, it is easily seen that

$$
(\mu \wedge v)+\left(\mu^{\prime} \wedge v^{\prime}\right)
$$

because

$$
(\mu \wedge v)(3)=\frac{1}{3}<(\mu \wedge v)(2)=\frac{1}{2},
$$


while

$$
\left(\mu^{\prime} \wedge \nu^{\prime}\right)(3)=\frac{1}{10}>\left(\mu^{\prime} \wedge \nu^{\prime}\right)(2)=\frac{1}{20} .
$$

Similarly, we can show by an example that in general $\mu \sim \mu^{\prime}$ and $\nu \sim v^{\prime}$ do not imply $\mu \vee \nu \sim \mu^{\prime} \vee \nu^{\prime}$.

The next example deals with the operation of sum.

EXAMPLE 3.2. Suppose that $\mu \sim v$ and $\mu^{\prime} \sim v^{\prime}$. Then it is not necessary that $\mu+\mu^{\prime} \sim v+v^{\prime}$.

Let $G$ be the group of integers $\mathbb{Z}$ under addition.

Let

$$
\begin{aligned}
& \mu(x)= \begin{cases}1, & x \in 6 \mathbb{Z}, \\
\frac{1}{2}, & x \in 2 \mathbb{Z} \backslash 6 \mathbb{Z}, \quad \mu^{\prime}(x)=\left\{\begin{array}{ll}
1, & x \in 6 \mathbb{Z}, \\
\frac{3}{4}, & \text { otherwise, }
\end{array} \quad x \in 2 \mathbb{Z} \backslash 6 \mathbb{Z},\right. \\
\frac{1}{10}, & \text { otherwise, }\end{cases} \\
& v(x)= \begin{cases}1, & x \in 6 \mathbb{Z}, \\
\frac{2}{3}, & x \in 3 \mathbb{Z} \backslash 6 \mathbb{Z}, \quad v^{\prime}(x)=\left\{\begin{array}{ll}
1, & x \in 6 \mathbb{Z}, \\
\frac{1}{2}, & \text { otherwise, }
\end{array} \quad x \in 3 \mathbb{Z} \backslash 6 \mathbb{Z},\right. \\
\frac{1}{6}, & \text { otherwise. }\end{cases}
\end{aligned}
$$

Then

$$
(\mu+v)(x)= \begin{cases}1, & x \in 6 \mathbb{Z}, \\
\frac{2}{3}, & x \in 3 \mathbb{Z} \backslash 6 \mathbb{Z}, \quad\left(\mu^{\prime}+\nu^{\prime}\right)(x)=\left\{\begin{array}{ll}
1, & x \in 6 \mathbb{Z}, \\
\frac{3}{4}, & \text { otherwise, }
\end{array} \quad x \in 2 \mathbb{Z} \backslash 6 \mathbb{Z},\right. \\
\frac{1}{2}, & \text { otherwise. }\end{cases}
$$

Firstly, we notice that

$$
\mu \sim \mu^{\prime}, \quad v \sim v^{\prime}
$$

Secondly, it is easily seen that

$$
(\mu+v) \ngtr\left(\mu^{\prime}+v^{\prime}\right)
$$

because

$$
(\mu+v)(3)=\frac{2}{3}>(\mu+v)(2)=\frac{1}{2},
$$

while

$$
\left(\mu^{\prime}+v^{\prime}\right)(3)=\frac{1}{2}<\left(\mu^{\prime}+v^{\prime}\right)(2)=\frac{3}{4} \text {. }
$$


NoTE 3.3. Although in this example $\mu+v \sim v$, this needs not be true in general. For example, if $1 / 6$ is replaced by 0 in both $v$ and $v^{\prime}$ above, then $\mu+\nu+\nu$ and $\mu+\nu+\mu$.

In contrast to the above examples, if we take two fuzzy subgroups $\mu$ and $v$ from the same equivalence class $\mathfrak{I}$ determined by $\mu$ and $\nu$, then the inf, sup, and sum of $\mu$ and $v$ determine the same equivalence class $\mathfrak{J}$.

Proposition 3.4. If $\mu \sim v$, then $\mu \wedge v \sim \mu$. Consequently, $\mu \wedge v \sim v$.

Proof. Since $\mu \sim \nu, \operatorname{supp} \mu=\operatorname{supp} v$, and also $\operatorname{supp}(\mu \wedge \nu)=\operatorname{supp} \mu \cap$ $\operatorname{supp} v$, we have $\operatorname{supp}(\mu \wedge v)=\operatorname{supp} \mu$. For the other part of equivalence, clearly, if $\mu(x)>\mu(y)$, then $v(x)>v(y)$ implies $(\mu \wedge v)(x)>(\mu \wedge v)(y)$.

Now, suppose that $(\mu \wedge v)(x)>(\mu \wedge v)(y)$.

CASE 1. Assume that $\mu(x)=(\mu \wedge v)(x)$ and $\mu(y)=(\mu \wedge v)(y)$, then there is nothing to prove.

CASE 2. If $\mu(x)=\mu \wedge v(x)$ and $v(y)=\mu \wedge v(y)$, then $v(x) \geq \mu(x)>v(y)$, which in turn implies $\mu(x)>\mu(y)$. Other cases are dealt with similarly.

Proposition 3.5. If $\mu \sim \nu$, then $\mu \vee v \sim \mu$. Consequently, $\mu \vee v \sim \nu$.

For the following proposition, we require both $\mu$ and $\nu$ to be fuzzy subgroups of a finite group.

Proposition 3.6. If $\mu \sim v$, then $\mu+v \sim \mu$. Consequently, $\mu+v \sim v$.

Proof. Suppose that

$$
(\mu+v)(x)>(\mu+v)(y)
$$

Then there are $x_{1}$ and $x_{2}$ with $x=x_{1}+x_{2}$ such that

$$
\mu\left(x_{1}\right) \wedge v\left(x_{2}\right)>(\mu+v)(y) .
$$

Suppose that $\mu\left(x_{1}\right)=\mu\left(x_{1}\right) \wedge \nu\left(x_{2}\right)$. Then $\mu\left(x_{1}\right)>\mu(y)$ and $\nu\left(x_{2}\right) \geq \mu\left(x_{1}\right)>$ $\nu(y)$. These imply $\mu\left(x_{2}\right)>\mu(y)$. Therefore, $\mu(x) \geq \mu\left(x_{1}\right) \wedge \mu\left(x_{2}\right)>\mu(y)$. Similarly, the other case $v\left(x_{2}\right)=\mu\left(x_{1}\right) \wedge \nu\left(x_{2}\right)$ leads to $\mu(x)>\mu(y)$.

Conversely, suppose that $\mu(x)>\mu(y)$. Then $(\mu+v)(x)>(\mu+v)(y)$ because if $(\mu+v)(x)<(\mu+v)(y)$, then by the above argument, we have $\mu(y)>$ $\mu(x)$, a contradiction. On the other hand, $(\mu+\nu)(x)=(\mu+v)(y)$ also leads to a contradiction in the following way: there exist $y_{1}$ and $y_{2}$ such that $(\mu+$ $v)(x)=\mu\left(y_{1}\right) \wedge v\left(y_{2}\right)$ and $y=y_{1}+y_{2}$. So, $\mu\left(y_{1}\right) \geq(\mu+v)(x) \geq \mu(x)$ and $\nu\left(y_{2}\right) \geq v(x)$ implying $\mu\left(y_{2}\right) \geq \mu(x)$. Thus, $\mu(y) \geq \mu\left(y_{1}\right) \wedge \mu\left(y_{2}\right) \geq \mu(x)$. This completes the proof.

But, as for Zadeh's complement [6], we have the following proposition. 
Proposition 3.7. If $\mu \sim v$, then $1-\mu \sim 1-v$.

4. Intersection and sums of fuzzy subgroups. In this section, we determine the equivalence class of fuzzy subgroups corresponding to intersection and sum of two fuzzy subgroups in terms of pinned flags associated with given fuzzy subgroups. Throughout this section, we require the number of components in a pinned flag to be at least 3, otherwise the discussions become trivial. Consequently, we assume that $n \geq 2$.

Firstly, in the next proposition, we look at a special case, namely the characterization of intersection and sum of two equivalence classes of fuzzy subgroups whose pinned flags $(\mathscr{C}, l)$ have the same underlying flag of subgroups (C).

Proposition 4.1. Let $G$ be a group with a flag $\mathscr{C}$ of subgroups $\{0\}=G_{0} \subset$ $G_{1} \subset G_{2} \subset \cdots \subset G_{n}=G$. Suppose that $\mu$ and $v$ are two fuzzy subgroups whose pinned flags have the same underlying flag $\mathscr{b}$ but have different keychains of the forms $\mu=1 \lambda_{1} \lambda_{2} \cdots \lambda_{n}$ and $v=1 \beta_{1} \beta_{2} \cdots \beta_{n}$, respectively. Then

(i) $\mu \wedge v \sim 1 \lambda_{1} \wedge \beta_{1} \cdots \lambda_{n} \wedge \beta_{n}$,

(ii) $\mu+v \sim 1 \lambda_{1} \vee \beta_{1} \cdots \lambda_{n} \vee \beta_{n}$.

NoTE 4.2. We emphasize in this note that $\mu$ and $v$ are not necessarily equivalent.

Proof. Let $x \in G$. Then there is an index $i$ such that $1 \leq i \leq n$ with $x \in G_{i}$, but $x \notin G_{i-1}$. Then $\mu(x)=\lambda_{i}$ and $\nu(x)=\beta_{i}$.

(i) To prove this part, it suffices to check that $(\mu \wedge v)(x)=\lambda_{i} \wedge \beta_{i}$, which is clearly true.

(ii) For this part, we first observe that $(\mu+\nu)(x) \geq \lambda_{i} \vee \beta_{i}$. Suppose that $(\mu+$ $v)(x)>\lambda_{i} \vee \beta_{i}$, then there exist $x_{1}$ and $x_{2}$ such that $x=x_{1}+x_{2}$ with $\mu\left(x_{1}\right) \wedge$ $v\left(x_{2}\right)>\lambda_{i} \vee \beta_{i}$. This implies $\mu\left(x_{1}\right)>\lambda_{i}$ and $v\left(x_{2}\right)>\beta_{i}$, which in turn implies $x_{1} \in G_{i-1}$ and $x_{2} \in G_{i-1}$. Thus, $x \in G_{i-1}$, a contradiction. This completes the proof.

Suppose that we have two flags differing in only one component, such as

$$
\begin{aligned}
& \mathscr{C}_{\mu}:\{0\}=G_{0} \subset G_{1} \subset G_{2} \subset \cdots \subset G_{k-1} \subset G_{k} \subset G_{k+1} \subset \cdots \subset G_{n}=G, \\
& \mathscr{C}_{v}:\{0\}=G_{0} \subset G_{1} \subset G_{2} \subset \cdots \subset G_{k-1} \subset H_{k} \subset G_{k+1} \subset \cdots \subset G_{n}=G,
\end{aligned}
$$

for $1 \leq k \leq n-1$.

Proposition 4.3. Suppose that $\mu$ and $v$ are fuzzy subgroups of $G$ whose representative keychains are of the form $1 \lambda_{1} \lambda_{2} \cdots \lambda_{n}$ and whose underlying flags are $\mathscr{C}_{\mu}$ and $\mathscr{C}_{\nu}$, respectively, as described above. Then

(i) $\mu \wedge v$ is represented by the keychain

$$
1 \lambda_{1} \lambda_{2} \cdots \lambda_{k-1} \lambda_{k+1} \lambda_{k+1} \cdots \lambda_{n} \quad \text { on } \mathscr{C}_{\mu} \text { or on } \mathscr{C}_{v}
$$


(ii) $\mu+v$ is represented by the keychain

$$
1 \lambda_{1} \lambda_{2} \cdots \lambda_{k-1} \lambda_{k} \lambda_{k} \lambda_{k+2} \cdots \lambda_{n} \text { on } \mathscr{C}_{\mu} \text { or on } \mathscr{b}_{v}
$$

Before we give the proof, we note that $\mathscr{C}_{\mu}$ can equivalently be replaced by $\mathscr{C}_{v}$ without any loss of generality.

PROOF. (i) It is straightforward from Proposition 4.1 that $(\mu \wedge v)(x)$ has the same keychain pins as $\mu$ and $v$ on all $G_{i}$ 's for $i=1,2, \ldots, k-1, k+1, \ldots, n$. It suffices to prove the case for $i=k$. So, consider $x \in G_{k+1} \backslash G_{k-1}$. Then $(\mu \wedge v)(x) \geq \lambda_{k+1}$. Suppose that $(\mu \wedge v)(x)>\lambda_{k+1}$. Then either $(\mu \wedge v)(x)=\lambda_{s}$ for some $1 \leq s \leq k$ or $(\mu \wedge \nu)(x)=1$. The latter case implies $\mu(x)=1=v(x)$. But, $x \notin G_{k-1}$ implies $\mu(x)<\lambda_{k-1}$. This in turn implies $\mu(x) \neq 1$, a contradiction. For the former case, $\lambda_{s} \geq \lambda_{k}$, implying $\mu(x) \geq \lambda_{k}$, which implies $x \in G_{k}$. Similarly, $x \in H_{k}$. So, $x \in G_{k} \cap H_{k}$. By the maximality of the chains involved, $G_{k} \cap H_{k}=G_{k-1}$. This implies $x \in G_{k-1}$, which is a contradiction to the choice of $x$. Thus, $(\mu \wedge v)(x)=\lambda_{k+1}$ for all $x \in G_{k+1} \backslash G_{k-1}$.

(ii) Firstly, we observe that $G_{k+1}=G_{k}+H_{k}$ by the maximality of chains. Secondly, similar to the proof of case (i), for the sum, it is enough to consider $x \in G_{k+1} \backslash G_{k-1}$. Then $(\mu+v)(x) \geq \mu\left(x_{1}\right) \wedge \nu\left(x_{2}\right) \geq \lambda_{k}$ for $x=x_{1}+x_{2}$ with $x_{1} \in G_{k}$ and $x_{2} \in H_{k}$. As before, suppose that $\mu\left(x_{1}\right) \wedge v\left(x_{2}\right)>\lambda_{k}$. Then either $\mu\left(x_{1}\right) \wedge \nu\left(x_{2}\right)=\lambda_{s}$ for some $1 \leq s \leq k-1$ or $\mu\left(x_{1}\right) \wedge \nu\left(x_{2}\right)=1$. The latter case implies $x=x_{1}+x_{2} \in G_{k-1}+G_{k-1}=G_{k-1}$, and in the former case, $x=x_{1}+x_{2} \in$ $G_{s}+G_{s}=G_{s}$. By the chain property, we conclude that $x \in G_{k-1}$, which is a contradiction to the choice of $x$. Thus, $(\mu+\nu)(x)=\lambda_{k}$ for all $x \in G_{k+1} \backslash G_{k-1}$. This completes the proof.

Suppose that we have two flags differing in two or more components but not consecutively, such as

$$
\begin{aligned}
& \mathscr{C}_{\mu}:\{0\}=G_{0} \subset G_{1} \subset \cdots \subset G_{i_{1}} \subset \cdots \subset G_{i_{2}} \subset \cdots \subset G_{i_{k}} \subset \cdots \subset G_{n}=G, \\
& \mathscr{C}_{v}:\{0\}=G_{0} \subset G_{1} \subset \cdots \subset H_{i_{1}} \subset \cdots \subset H_{i_{2}} \subset \cdots \subset H_{i_{k}} \subset \cdots \subset G_{n}=G,
\end{aligned}
$$

where

$$
1 \leq i_{1}<i_{1}+2 \leq i_{2}<i_{2}+2 \leq \cdots<i_{k-1}<i_{k-1}+2 \leq i_{k} \leq n-1 .
$$

Then, using the same argument as in Proposition 4.3 inductively, we have the following corollary.

COROLLARY 4.4. Let $\mu$ and $v$ be two fuzzy subgroups whose underlying flags $\mathscr{C}_{\mu}$ and $\mathscr{C}_{v}$, respectively, differ in two or more components but not consecutively as shown in (4.4). Then

(i) $\mu \wedge v$ is represented by the keychain

$$
1 \lambda_{1} \cdots \lambda_{i_{1}-1} \lambda_{i_{1}+1} \lambda_{i_{1}+1} \cdots \lambda_{i_{k}-1} \lambda_{i_{k}+1} \lambda_{i_{k}+1} \cdots \lambda_{n} \quad \text { on } \mathscr{C}_{\mu} \text { or on } \mathscr{C}_{v}
$$


(ii) $\mu+v$ is represented by the keychain

$$
1 \lambda_{1} \cdots \lambda_{i_{1}-1} \lambda_{i_{1}} \lambda_{i_{1}} \cdots \lambda_{i_{k}-1} \lambda_{i_{k}} \lambda_{i_{k}} \cdots \lambda_{n} \quad \text { on } \mathscr{C}_{\mu} \text { or on } \mathscr{C}_{v} .
$$

Now, we would like to consider two flags differing in two or more components consecutively, such as

$$
\begin{aligned}
& \mathscr{b}_{\mu}: \cdots \subset G_{i-1} \subset G_{i} \subset G_{i+1} \subset \cdots \subset G_{i+k-1} \subset G_{i+k} \subset G_{i+k+1} \subset \cdots, \\
& \mathscr{b}_{v}: \cdots \subset G_{i-1} \subset H_{i} \subset H_{i+1} \subset \cdots \subset H_{i+k-1} \subset H_{i+k} \subset G_{i+k+1} \subset \cdots,
\end{aligned}
$$

for $1 \leq i<\cdots<i+k \leq n-1, k \geq 1$. Then the flags $\mathscr{C}_{\mu \wedge \nu}$ and $\mathscr{C}_{\mu+\nu}$ are given by

$$
\mathscr{C}_{\mu \wedge v}: \cdots \subset G_{i-1} \subset G_{i+1} \cap H_{i+1} \subset \cdots \subset G_{i+k} \cap H_{i+k} \subset F \subset G_{i+k+1} \subset \cdots,
$$

where $F$ can be either $G_{i+k}$ or $H_{i+k}$, and

$$
\mathscr{C}_{\mu+v}: \cdots \subset G_{i-1} \subset E \subset G_{i}+H_{i} \subset \cdots \subset G_{i+k-1}+H_{i+k-1} \subset G_{i+k+1} \subset \cdots,
$$

where $E$ can be either $G_{i}$ or $H_{i}$, respectively.

In the above, we have only indicated the corresponding distinct components in $\mathscr{b}_{\mu}, \mathscr{b}_{\nu}, \mathscr{b}_{\mu \wedge \nu}$, and $\mathscr{b}_{\mu+\nu}$ and as the suppressed corresponding components are assumed to be identical in the two flags.

Proposition 4.5. Suppose that $\mu$ and $v$ are fuzzy subgroups of $G$ whose representative keychains are of the form $1 \lambda_{1} \lambda_{2} \cdots \lambda_{n}$ and whose underlying flags are $\mathfrak{C}_{\mu}$ and $\mathscr{C}_{\nu}$, respectively, as described above. Then

(i) $\mu \wedge \nu$ is represented by the keychain

$$
1 \lambda_{1} \lambda_{2} \cdots \lambda_{i-1} \lambda_{i+1} \lambda_{i+2} \cdots \lambda_{i+k} \lambda_{i+k+1} \lambda_{i+k+1} \lambda_{i+k+2} \cdots \lambda_{n} \quad \text { on } \mathscr{C}_{\mu \wedge v},
$$

(ii) $\mu+v$ is represented by the keychain

$$
1 \lambda_{1} \lambda_{2} \cdots \lambda_{i-1} \lambda_{i} \lambda_{i} \lambda_{i+1} \cdots \lambda_{i+k} \lambda_{i+k+2} \cdots \lambda_{n} \quad \text { on } \mathscr{C}_{\mu+v}
$$

Proof. As in Proposition 4.3, it suffices to consider only indices $i, i+1, \ldots$, $i+k$.

(i) Let $x \in G_{i+1} \cap H_{i+1} \backslash G_{i-1}$. Then clearly $\lambda_{i-1}>(\mu \wedge v)(x) \geq \lambda_{i+1}$. Now, suppose that $(\mu \wedge v)(x)>\lambda_{i+1}$. Then $(\mu \wedge v)(x)=\lambda_{i}$ implying $x \in G_{i} \cap H_{i}=$ $G_{i-1}$ by maximality of $\mathscr{C}_{\mu}$ and $\mathscr{C}_{\nu}$. This is a contradiction to our choice of $x$. Hence, $(\mu \wedge v)(x)=\lambda_{i+1}$.

Now, for other cases, let $x \in G_{i+j} \cap H_{i+j} \backslash G_{i+j-1} \cap H_{i+j-1}$ for $j=2,3, \ldots, k$. Then $\lambda_{i+j-1}>(\mu \wedge v)(x) \geq \lambda_{i+j}$. Suppose that $(\mu \wedge v)(x)>\lambda_{i+j}$. Then there exists a pin $\lambda_{s}$ representing the value of $(\mu \wedge \nu)(x)$ and it is such that $\lambda_{i+j-1}>$ $\lambda_{s}>\lambda_{i+j}$, which is a contradiction as $\lambda_{i+j-1}$ and $\lambda_{i+j}$ are two consecutive pins. Thus, $(\mu \wedge v)(x)=\lambda_{i+j}$. 
Next, let $x \in F \backslash G_{i+k} \cap H_{i+k}$, say $F=H_{i+k}$. Then $\nu(x) \geq \lambda_{i+k}$ and $\mu(x)<\lambda_{i+k}$, which implies $(\mu \wedge v)(x)=\mu(x)<\lambda_{i+k}$. But, $(\mu \wedge v)(x) \geq \lambda_{i+k+1}$. Suppose that $(\mu \wedge \nu)(x)>\lambda_{i+k+1}$. Then this leads to a contradiction as in the previous case. Thus, $(\mu \wedge \nu)(x)=\lambda_{i+k+1}$. Similarly, if $F=G_{i+k}$.

Finally, let $x \in G_{i+k+1} \backslash F$, say $F=H_{i+k}$. Then $(\mu \wedge v)(x) \geq \lambda_{i+k+1}$ and $v(x)<$ $\lambda_{i+k}$, which implies $(\mu \wedge \nu)(x)<\lambda_{i+k}$. As in the previous cases, we conclude that $(\mu \wedge \nu)(x)=\lambda_{i+k+1}$. Similarly, for $F=G_{i+k}$. Thus (i) is proved.

(ii) Let $x \in E \backslash G_{i-1}$, say $E=H_{i}$. Then $v(x) \geq \lambda_{i}$ implying $(\mu+v)(x) \geq \lambda_{i}$. Suppose that $(\mu+\nu)(x) \geq \lambda_{i-1}$. Then $x \in G_{i-1}$, a contradiction. Thus, $\lambda_{i} \leq$ $(\mu+v)(x)<\lambda_{i-1}$. As in case (i) above, we conclude that $(\mu+v)(x)=\lambda_{i}$.

Next, let $x \in G_{i}+H_{i} \backslash E$, say $E=H_{i}$. Then $(\mu+\nu)(x) \geq \lambda_{i}$ and $\nu(x)<\lambda_{i}$. Suppose that $(\mu+\nu)(x) \geq \lambda_{i-1}$. Then $x \in G_{i-1} \subseteq H_{i}$, a contradiction. Thus, $\lambda_{i} \leq(\mu+v)(x)<\lambda_{i-1}$. Hence, as before, $(\mu+v)(x)=\lambda_{i}$. Similarly, if $E=G_{i}$.

Now, for other cases, let $x \in G_{i+j}+H_{i+j} \backslash G_{i+j-1}+H_{i+j-1}$ for $j=1,2, \ldots, k-1$. Then $\lambda_{i+j-1}>(\mu+v)(x) \geq \lambda_{i+j}$. As in other parts of this proof, it is clear that $(\mu+v)(x)=\lambda_{i+j}$.

Finally, let $x \in G_{i+k+1} \backslash G_{i+k-1}+H_{i+k-1}$. Then $(\mu+v)(x) \geq \lambda_{i+k+1}$. But, $G_{i+k+1}=$ $G_{i+k}+H_{i+k}$ by maximality of the chains $\mathscr{C}_{\mu}$ and $\mathscr{C}_{\nu}$. So, $\lambda_{i+k-1}>(\mu+\nu)(x) \geq$ $\lambda_{i+k}$. Thus, $(\mu+\nu)(x)=\lambda_{i+k}$. This completes the proof.

The determination of the pinned flags of intersection and sum of two fuzzy subgroups $\mu$ and $\nu$, where the pins as well as the flags of the pinned flags $\mathscr{C}_{\mu}$ and $\mathscr{C}_{\nu}$ representing $\mu$ and $\nu$ are distinct, in general, does not seem to follow any particular pattern as we have derived above. This is illustrated by the following example.

EXAMPLE 4.6. Let $G=\mathbb{Z}_{72}$. Let $\mathscr{C}_{\mu}$ and $\mathscr{C}_{\nu}$ be the pinned flags of $\mu$ and $\nu$ on $G$ given by

$$
\begin{aligned}
& \mathscr{C}_{\mu}: 0^{1} \subset \mathbb{Z}_{3}{ }^{1 / 2} \subset \mathbb{Z}_{9}{ }^{1 / 5} \subset \mathbb{Z}_{18}{ }^{1 / 6} \subset \mathbb{Z}_{36}{ }^{1 / 9} \subset \mathbb{Z}_{72}{ }^{1 / 10}, \\
& \mathscr{C}_{v}: 0^{1} \subset \mathbb{Z}_{3}{ }^{1 / 3} \subset \mathbb{Z}_{6}{ }^{1 / 4} \subset \mathbb{Z}_{12}{ }^{1 / 7} \subset \mathbb{Z}_{36}{ }^{1 / 8} \subset \mathbb{Z}_{72}{ }^{1 / 11},
\end{aligned}
$$

respectively. A simple calculation reveals the pinned flags for $\mu \wedge \nu$ and $\mu+\nu$ to be

$$
\begin{aligned}
& 0^{1} \subset \mathbb{Z}_{3}{ }^{1 / 3} \subset \mathbb{Z}_{6}{ }^{1 / 6} \subset \mathbb{Z}_{18}{ }^{1 / 8} \subset \mathbb{Z}_{36}{ }^{1 / 9} \subset \mathbb{Z}_{72}{ }^{1 / 11}, \\
& 0^{1} \subset \mathbb{Z}_{3}{ }^{1 / 2} \subset \mathbb{Z}_{6}{ }^{1 / 4} \subset \mathbb{Z}_{18}{ }^{1 / 5} \subset \mathbb{Z}_{36}{ }^{1 / 7} \subset \mathbb{Z}_{72}{ }^{1 / 10},
\end{aligned}
$$

respectively. In the above calculation, notice that the roles played by the pins and the components of the flags are equally important in a way in which they are tied to each other. Suppose that we retain the flags but the pins for $\mu$ and $v$ are changed to

$$
1 \frac{1}{3} \frac{1}{9} \frac{1}{18} \frac{1}{36} \frac{1}{72}, \quad 1 \frac{1}{72} \frac{1}{90} \frac{1}{100} \frac{1}{110} \frac{1}{120},
$$


respectively. Then $\mu \wedge \nu$ and $\mu+\nu$ have the pinned flags given by

$$
\begin{aligned}
& 0^{1} \subset \mathbb{Z}_{3}{ }^{1 / 72} \subset \mathbb{Z}_{6}{ }^{1 / 90} \subset \mathbb{Z}_{12}{ }^{1 / 100} \subset \mathbb{Z}_{36}{ }^{1 / 110} \subset \mathbb{Z}_{72}{ }^{1 / 120}, \\
& 0^{1} \subset \mathbb{Z}_{3}{ }^{1 / 3} \subset \mathbb{Z}_{9}{ }^{1 / 9} \subset \mathbb{Z}_{18}{ }^{1 / 18} \subset \mathbb{Z}_{36}{ }^{1 / 36} \subset \mathbb{Z}_{72}{ }^{1 / 72},
\end{aligned}
$$

respectively. Similarly, we could retain the pins but change the flags of $\mu$ and $v$; for example, in (4.13) above we retain the same pins but swop the underlying flags. Then a simple calculation shows that we arrive at different (from (4.14)) pinned flags for $\mu \wedge \nu$ and $\mu+v$. The complete determination of the pinned flags for $\mu$ and $v$ in the most general case will be dealt with in another paper.

ACKNOWLEDGments. We thank both the NRF and the JRC of Rhodes University for financial support, which enabled us to travel between the University of Fort Hare and Rhodes University on a regular weekly basis to complete this work. Further, we thank Greg Lubczonok for some useful conversation on this topic.

\section{REFERENCES}

[1] J. B. Fraleigh, A First Course in Abstract Algebra, Addison-Wesley, Massachusetts, 1982.

[2] G. Lubczonok and V. Murali, On flags and fuzzy subspaces of vector spaces, Fuzzy Sets and Systems 125 (2002), no. 2, 201-207.

[3] V. Murali and B. B. Makamba, On an equivalence of fuzzy subgroups. I, Fuzzy Sets and Systems 123 (2001), no. 2, 259-264.

[4] _ On an equivalence of fuzzy subgroups II, Fuzzy sets and Systems 136 (2003), no. 1, 93-104.

[5] P. Sivaramakrishna Das, Fuzzy groups and level subgroups, J. Math. Anal. Appl. 84 (1981), no. 1, 264-269.

[6] L. A. Zadeh, Fuzzy sets, Information and Control 8 (1965), 338-353.

V. Murali: Department of Mathematics (Pure and Applied), Faculty of Science, Rhodes University, Grahamstown 6140, South Africa

E-mail address: v.murali@ru.ac.za

B. B. Makamba: Department of Mathematics, Faculty of Science, University of Fort Hare, Alice 5700, South Africa

E-mail address: bmakamba@ufh.ac.za 


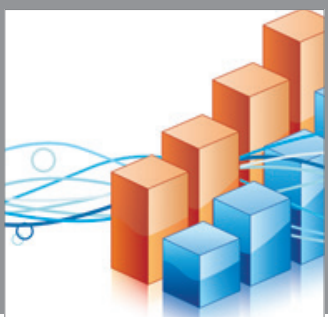

Advances in

Operations Research

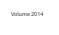

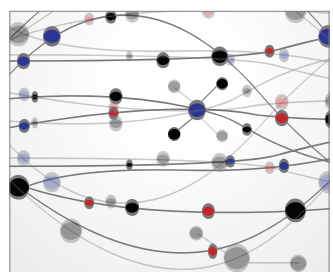

\section{The Scientific} World Journal
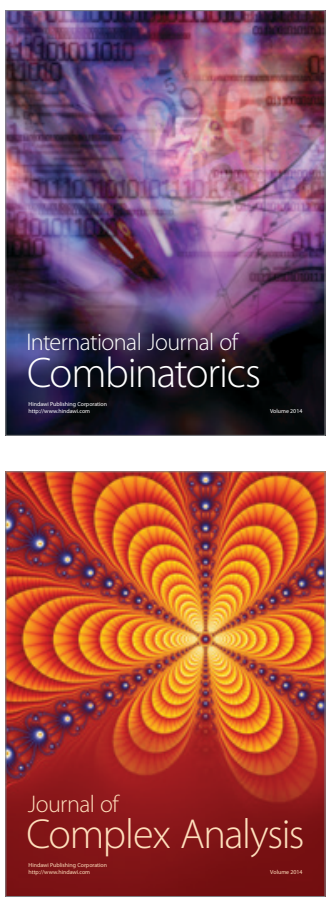

International Journal of

Mathematics and

Mathematical

Sciences
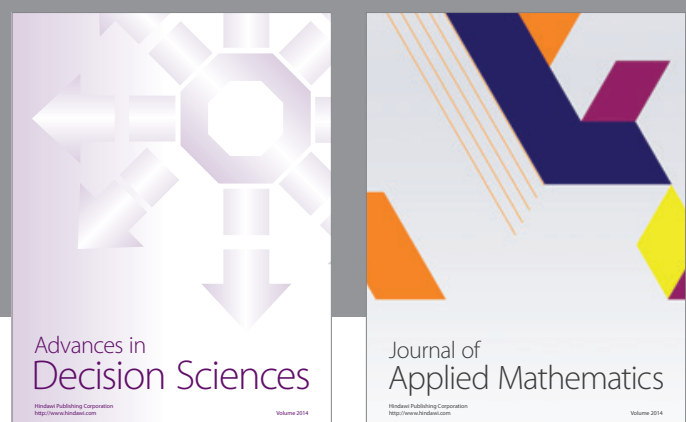

Journal of

Applied Mathematics
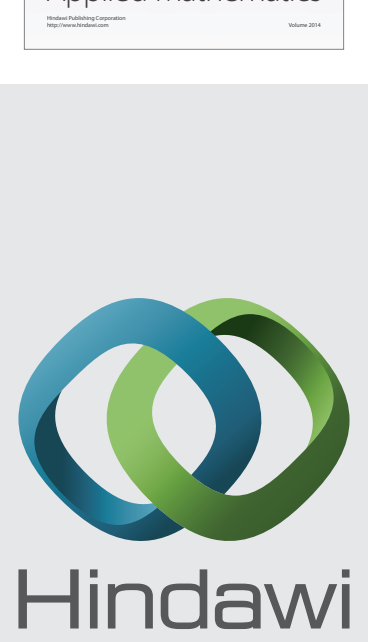

Submit your manuscripts at http://www.hindawi.com
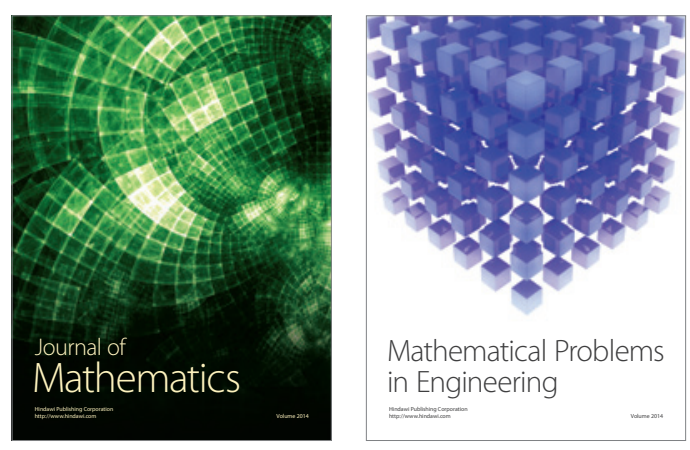

Mathematical Problems in Engineering
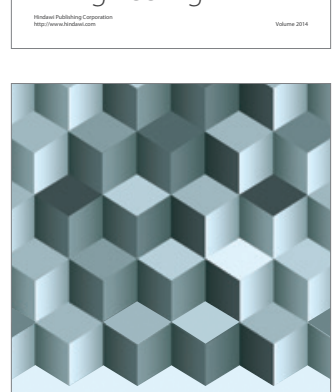

Journal of

Function Spaces
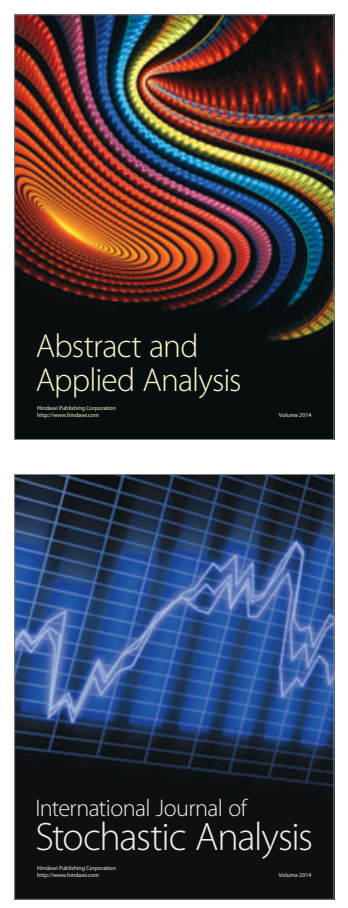

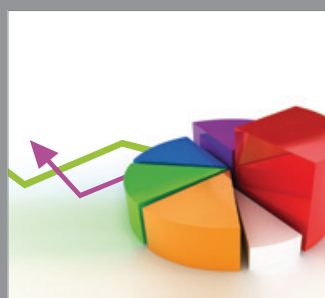

ournal of

Probability and Statistics

Promensencen
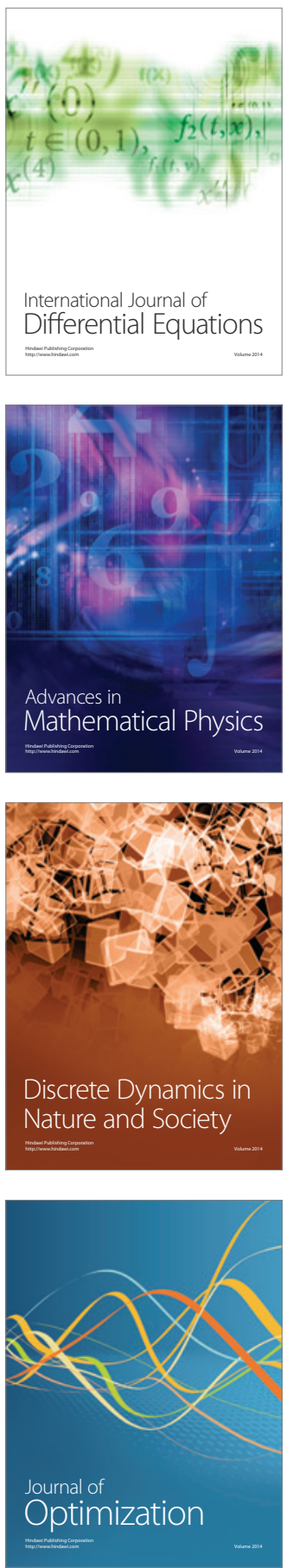